\title{
Communication between Outdoor Field and Immersive Virtual Environment
}

\author{
Tetsuro Ogi \\ University of Tsukuba \\ tetsu@cc.tsukuba.ac.jp
}

\author{
Tetsuro Fujise \\ Mitsubishi Research Institute, Inc. \\ fujise@mri.co.jp
}

\begin{abstract}
This paper proposes the concept of the hybrid information space that integrates the information space seamlessly between the real world and the virtual world, and the prototype system was developed. In this system, the users can communicate with each other between the real world and the virtual world or they can achieve their work while going and coming between both worlds. This system was applied to relive travel experiences and to assist campus navigation, and the effectiveness of this system was evaluated.
\end{abstract}

\section{Introduction}

Recent advances in cellular phones mean that they not only function as telephones, but can now also include various information technologies such as internet access, digital cameras, GPS; an electronic compass can even be installed; i.e. cellular phones are now used as highly-efficient portable information devices. By using these functions, a user can access various kinds of information freely at anytime and anywhere. However, in these devices, the level of detail in the information that is being represented is limited because the display window is small.

On the other hand, immersive projection displays such as CAVE or CABIN can display information with an abundance of detail by using large screens with wide fields of view and stereo vision functions [1]. However, in the case of these static displays, opportunities for using them are limited because users must go to the places where the display systems are located in order to experience any information that is being presented.

These two kinds of devices are entirely different from each other in terms of both features and usage, and they have usually been thought of as opposing devices. However, it is expected that a more effective information-access and presentation technology might be constructed by mutually integrating these devices so that they complement each other. In particular, communication environment would be generated by connecting these techniques through the network.

This study aims at constructing a hybrid information space in which the real world and the virtual world are connected seamlessly. Then, a prototype system was developed by synthesizing an information acquisition technique using a cellular phone in the outdoors field along with a high-presence visualization technique that uses an immersive projection display. In this system, the user can experience the hybrid information space while coming and going in both the real world and in the virtual world. Moreover, remote users can communicate with each other while sharing the high presence information between both worlds.

\section{Concept of Hybrid Information Space}

In the real world, we acquire a large amount of information from our surroundings through experience. Since this information is obtained through our senses, we could not experience it repeatedly without recording it with a high quality of presence. On the other hand, when we use a computer, we can repeatedly retrieve and use information that has been classified and stored in the database system. In particular, we can experience an information space by using our senses when it is visualized in a virtual world.

Hybrid information space is a concept that involves the seamless transfer of information between the real world and the virtual world. In this environment, the user can seamlessly experience the information that is acquired in the real world, as well as artificial information that is represented in the virtual world. For example, a user can retrieve required information from a database in the real world, or he can also refer to visualized data that was taken into the virtual world from the real world. This study aims at integrating both of these worlds so that the users can perform their works while swapping seamlessly between them or communicating between these worlds as shown in Figure 1. 


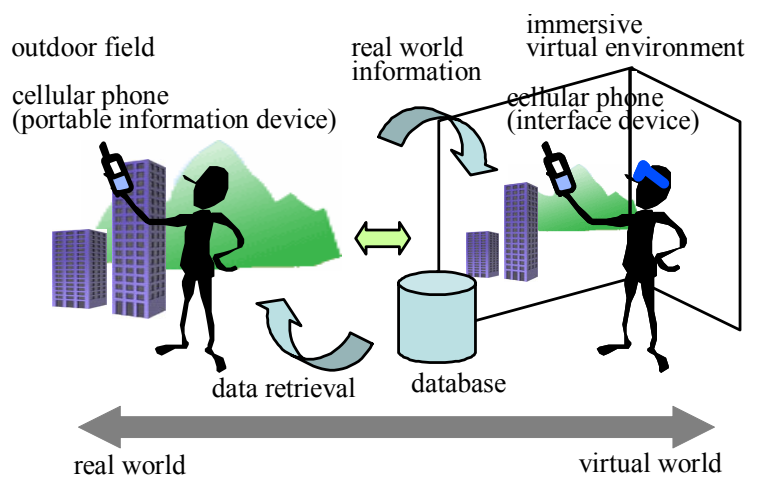

Figure1. Concept of hybrid information space

In the case of data acquisition using a cellular phone in the real world, several services have already been made available. In this study, a method of importing real-world information into an immersive virtual environment and of constructing a virtual space was examined in detail. This method enables the user to relive an experience in the virtual world after experiencing it in the field by representing highpresence information in an immersive projection environment. In addition, this method realizes highpresence communication between the users in the outdoor field and in the virtual world. In these cases, positional information obtained by the GPS is used as a key technology for integrating real world information into the virtual world. And, a cellular phone is used as the interface device as well as for the communication device.

\section{Prototype System}

\subsection{Information Acquisition in Outdoor Field}

In this study, we have developed a prototype system for experiencing hybrid information space. First, in order to record the information that the user experienced in the outdoor field, a cellular phone was used. Recently-developed cellular phones can provide various information access functions, and they have been used as highly-efficient portable information devices. In our experimental system, the Sony Ericsson W21S cellular phone that was equipped for the e-mail, the web access, the digital camera, the GPS and the electronic compass functions was used.

When the user wants to record a scene outdoors, he can take a picture of it using the digital camera function of the cellular phone as shown in Figure 2. At the same time, he can record data regarding the position and direction of the area where he is taking the picture by using the GPS and the electronic compass functions.

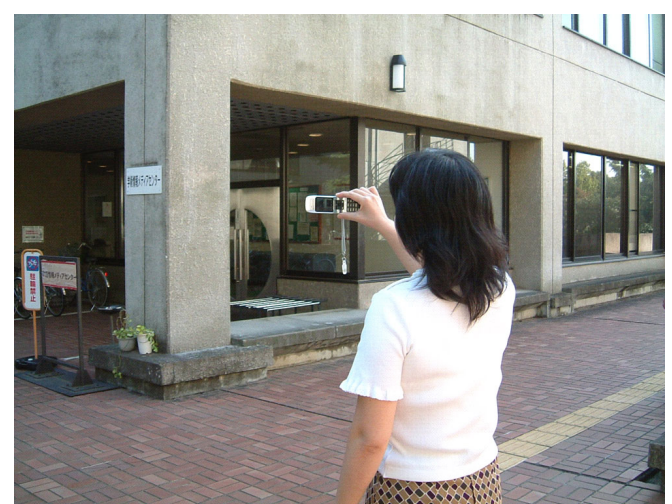

Figure2. Taking pictures in the outdoor field

Next, this information is sent to a server computer that is used to generate the virtual world by using the e-mail function. In this case, the photograph image is sent as an attached file, and the positional data for latitude and longitude measured by the GPS are inserted in the text column of the e-mail. Moreover, data concerning the direction of the camera, such as "north" or "south-west" were measured by the electronic compass and the rough distance to the scene, such as "short distance", "middle distance" or "long distance", were measured by observation, and these were also written in the text column. The use of "short distance" implies taking a picture of a human or a small object, "middle distance" usually means a building has been photographed, while "long distance" would typically mean that a panoramic scene has been shot. In addition, the user can also write an explanation or impression of the scene in the text column, so that this text information can be used as an annotation to the photographic image.

On the other hand, a graphics computer is used as the mail server, and when it receives the e-mail from the user in the outdoor field, a parser program analyzes the data in the e-mail message and stores each data item, such as the photographic image, the position (latitude and longitude), the direction, the distance, and any annotation in the database system. Figure 3 shows an example of data that is stored in the database system.

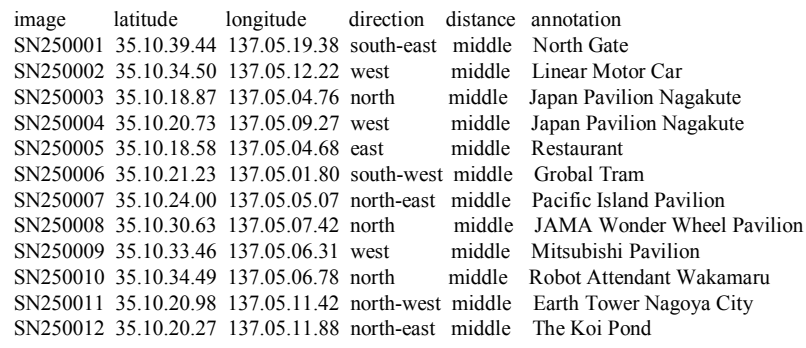

Figure3. Example of the transmitted data 


\subsection{Experience in Immersive Virtual World}

In order to visualize the virtual world, an immersive projection display named C.C. Wall was used. C.C. Wall is a stereo screen with dimensions of $2.0 \mathrm{~m} \mathrm{x}$ $2.5 \mathrm{~m}$, and the user can interact with the virtual world using a cellular phone interface [2]. This method utilizes the web-access function of the cellular phone. The user accesses a web page that has been prepared for the virtual reality application and communicates with the virtual world through the CGI program. In this case, the interaction menu is displayed on the cellular phone in the form of a web page, and the user selects commands from the listed items or inputs character commands using the dial button. Though this method includes some time delay owing to the extra communication through the cellular phone carrier, the user can easily input commands using the displayed interaction menu, not in three-dimensional virtual space, but on the LCD panel in his hand. Figure 4 shows an example of an interaction menu that might be displayed on the cellular phone. In this example, the user walks through the virtual world by pushing the dial button that corresponds to the moving operation displayed on the LCD. Thus, the user can bring the personal device of the cellular phone that was used to take pictures in the outdoor field into the immersive virtual environment, and can therefore seamlessly relive the experience between both worlds.

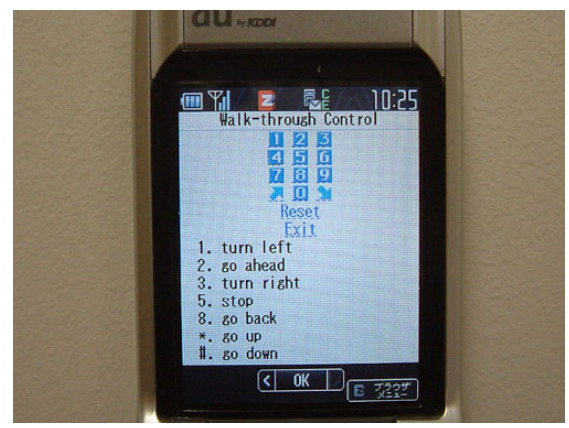

Figure4. Example of the Interaction menu

In this system, an 'empty world' is prepared as the initial state of the virtual world, in which only a texture image of a map was placed on the floor. The application program then retrieves the necessary information from the database, and places the photograph images and the annotation data at the corresponding positions according to the latitude, the longitude, the direction and the distance data. In this case, these photographic images are placed at $1 \mathrm{~m}, 20 \mathrm{~m}$, and $40 \mathrm{~m}$ away from the position of the camera in the virtual world, according to the distance data of "short distance", "middle distance" and "long distance" respectively, in locations where it can be seen at the same viewing angle from the camera position, as shown in Figure 5.

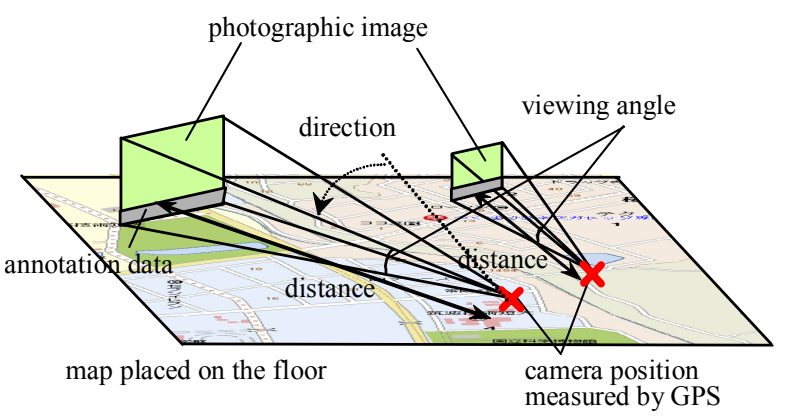

Figure5. Photograph based virtual world

Although the positions and the sizes of the visualized data are not strictly correct, they can be used to construct an approximated model of the virtual world, because the purpose of this study is not to construct a precise virtual world but to represent the spatial relationship among the information recorded in the real world. Thus, a virtual world of the information space can be constructed by placing a large amount of photographic images and annotation data on the blank map. Figure 6 shows an example in which the user is experiencing the virtual space constructed using the above-mentioned method. In this method, the user can relive the experience of the outdoor field in an approximated three-dimensional virtual world displayed in the C.C. Wall display. In particular, it is expected that this system could be effectively used to enable the user to construct a mental map and to memorize his experience from the spatial information.

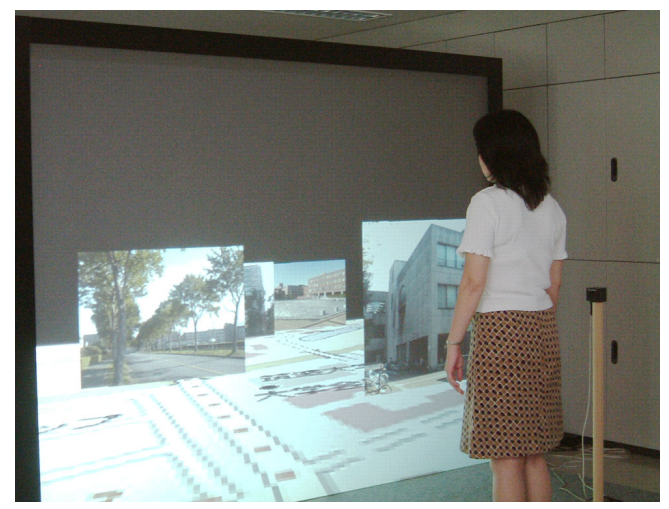

Figure6. Experience of the virtual world

\subsection{Communication between Real World and Virtual World}


In the above-mentioned scenario, the user relived a previously experienced space in an immersive virtual environment after experiencing it in the real world. This system can also be used for the communication between the real world and the virtual world by applying it in real-time. In this case, it is assumed that one user is experiencing the outdoor field and another user is in an immersive virtual environment.

When the user in the field sends a picture image and annotation data to the server computer, these data are stored in the database system in real-time. The application program checks the contents of the data in the database system at regular intervals, and updates the virtual world by adding any new data. By using this system, a user in the virtual world can recognize what the other user is experiencing in the real world, and can share part of the experience. In addition, the user can easily talk to the other user by using the cellular phone that is used as the interface device in both environments. Therefore, it has become possible for users in the outdoor field and in the immersive virtual world to communicate with each other while sharing the hybrid information space. Communication using hybrid information space can be effectively applied to experiencing virtual travel with a friend who is actually traveling in remote places or to providing remote instruction to a collaborator who is working outdoors in the field.

\section{Experiments Using Hybrid Information Space}

\subsection{Reliving Travel Experiences}

In this study, several experiments were conducted using a prototype system to investigate effective usages of hybrid information space. The reliving of travel experiences in the immersive virtual world is thought to be effective for firmly memorizing precious experiences. In the first experiment, the system was applied to recording travel experiences while visiting the World Expo held in Aichi in 2005. In this Expo, more than 100 pavilions were exhibited in an area of 1.82 square kilometers and visitors had to walk around many exhibition sites in a hurry in a limited time. Therefore, it was difficult for many visitors to understand the spatial information of the positional relationship between the exhibitions, though they felt a strong impression from each pavilion.

The subjects were asked to take pictures of those scenes where they gained a strong impression while walking through the Expo site by using the digital camera in the cellular phone, and then to send them to the server computer with the position and the annotation data. The subjects were also asked to experience a virtual world of the Expo that was constructed using the transmitted pictures in the C.C. Wall display after coming home from the actual Expo. Figure 7 shows an example of the virtual world that was constructed using the photograph images of the Expo site.

From the results, the subject recounted to us his impression that he could clearly memorize spatial relationships that had not been obtained during the real experience by reliving it in the immersive virtual world. Moreover, the information in the constructed virtual world was more and more enriched when several subjects performed the experimental task. Therefore, the hybrid information space is also useful for a user who experiences the virtual world before visiting the actual Expo, since he can gain prior knowledge and acquire a mental map of the Expo site.

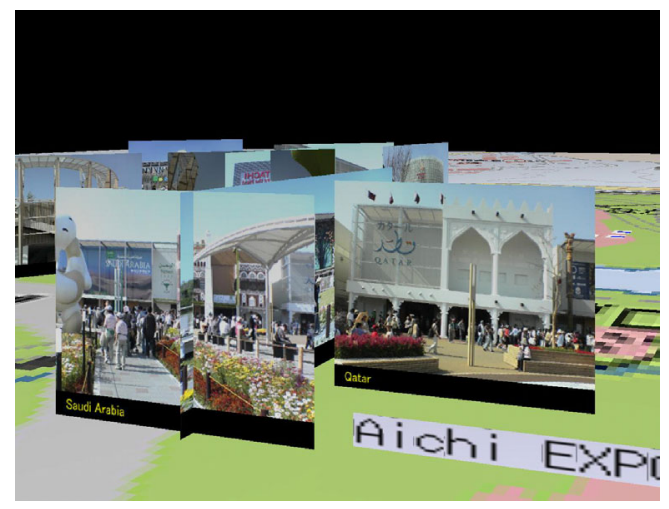

Figure7. Virtual world of the Expo site

\subsection{Campus Navigation}

Next, we conducted an experiment in which we applied this system to high-presence communication between the real world and the virtual world. In this experiment, a virtual world of our university campus was constructed beforehand using photographic images as shown in Figure 8, and it was utilized as a navigation aid for visitors, who used the real-time communication function. Since the University of Tsukuba has a large campus that is $4 \mathrm{~km}$ long from south-to-north and is $1 \mathrm{~km}$ wide from east-to-west, visitors often loose their ways when traveling around it. This experiment was conducted to evaluate whether visitors could be effectively navigated to their destination using this system.

When we had a call from the visitor who was participating in the experiment, we asked him to send a photographic image and positional data about the place 
where he was located to the server computer. These transmitted data were added to the virtual world in real-time. Then the navigator, who was in the immersive virtual world, was able to recognize where the visitor was located from the transmitted photographic images, and he navigated the visitor to the author's office by talking on the cellular phone through the immersive virtual world. In this experiment, since the navigator and the visitor share the visual information space of the university campus, the navigator was able to give instructions to the visitor while looking at the same world from the visitor's view point.

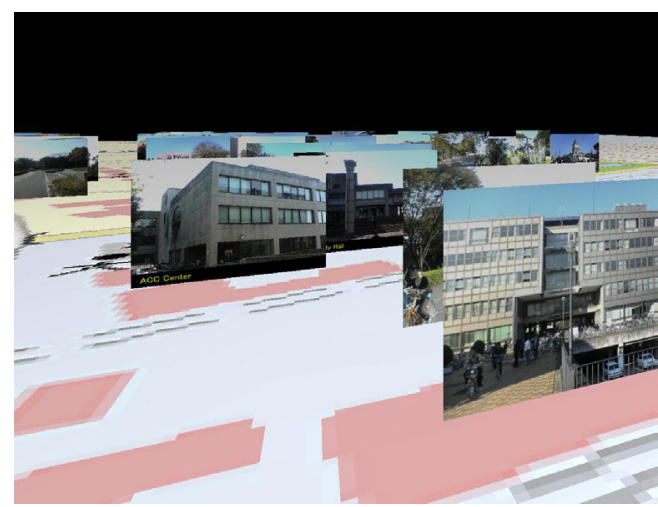

Figure8. Virtual world of the university campus

\section{Discussions}

Hybrid information space is a concept that seamlessly integrates information between the real world and the virtual world. Various kinds of high presence virtual reality systems using the photograph images have been developed [3][4]. Compared with these systems, this study aims at constructing an information space in which the users can communicate or come and go between the real world and the virtual world in order to achieve his work. Therefore, though the accuracy or the reality of the constructed virtual world is not so high due to the usage of twodimensional photographic images, it can be generated easily by using information that is gathered in the real world.

Though this prototype system only implemented functions for bringing information from the real world to the virtual world, the targeted hybrid information space aims at realizing mutual integration between both worlds by introducing a function to extract information from the virtual world to the real world. Several services in which a user in the real world can access a database by using a cellular phone with a GPS receiver are already available. However, with this system, it is expected that the user could not only refer to organized information stored in the database system, but also access un-compiled data such as annotation information that has been generated through experiences in the virtual world. Therefore, the results of prior experience in the immersive virtual world could be effectively used in real world experiences.

In addition, in the hybrid information space, a user can acquire necessary information while coming and going between the real world and the virtual world. This style of usage can be applied to various fields of application in which the user needs to access information. For example, experiences in the immersive virtual world could be used as an effective method of achieving prior and posterior learning for study tours or for school excursions.

\section{Conclusions}

In this study, the concept of a hybrid information space that integrates information from the real world with information from a virtual world was proposed, and a prototype system for constructing an immersive virtual world using the photographic images that were taken outdoors in the field was developed. This system can be used to relive experiences in the virtual world or to realize communication between users in the real world and in the virtual world. In our experiments, this system was applied to relive travel experiences and to assist in campus navigation, and it was found to be effective in both cases. In future work, we are planning to introduce a function whereby a user in the real world could access information that is generated in the virtual world, and to evaluate the effectiveness of mutual integration between both environments.

\section{References}

[1] M. Hirose, T. Ogi,, S. Ishiwata, T. Yamada, "Development and Evaluation of the Imersive Multiscreen Display CABIN", Systems and Computers in Japan, 1999, Vol.30, No.1, pp.13-22.

[2] T. Ogi, K. Yamamoto, T. Yamada, M. Hirose, "Experience of Virtual World Using Cellular Phone Interface”, Proc. of IEEE PCM 2001, 2001, pp.32-39.

[3] P. Milgram, H. Takemura, A. Utsumi, F. Kishino, "Augmented Reality: A Class of Displays on the RealityVirtuality Continuum. SPIE Vol. 2351, 1994, pp.282-292.

[4] T. Endo, A. Katayama, H. Tamura, M. Hirose, T. Tanikawa, "Cybercity Walker -Layered Morphing Method", Proc. of HCI'99, 1999, pp.1044-1048. 